\title{
Computational approaches to robust Model Predictive Control: a comparative analysis. ${ }^{\star}$
}

\author{
Luca Deori* Simone Garatti* Maria Prandini* \\ * Dipartimento di Elettronica, Informazione e Bioingegneria, \\ Politecnico di Milano, Piazza Leonardo da Vinci 32, 20113 Milano, \\ Italy. E-mail: \{deori, sgaratti,prandini\}@elet.polimi.it
}

\begin{abstract}
Robust Model Predictive Control (MPC) is a powerful technique to deal with constrained control of systems subject to bounded uncertainty. In the literature, many approaches have been proposed to find computationally feasible solutions. In this paper, we focus on robust MPC for linear systems affected by additive disturbances. Our aim is that of presenting a thorough comparison between two types of computational approaches: those that provide a solution that is robustly guaranteed, and randomized-based methods, which provide a solution with probabilistic guarantees only. The main outcome of the analysis is that the first type of approaches may show some conservatism in that they require the constraints to be loose in order to be feasible, while, although robustness cannot be a-priori guaranteed, randomized methods may lead to an effective solution - whose robustness can be experimentally verified - even for tight constraints. This enhances the use of randomized-based methods as a valid alternative to other approaches to robust MPC.
\end{abstract}

\section{INTRODUCTION AND PROBLEM SETUP}

Robust Model Predictive Control (MPC) is a powerful technique to deal with constrained control of systems subject to bounded uncertainty. In the literature, many approaches have been proposed to find computationally feasible solutions. In this paper, we focus on robust MPC for linear systems affected by additive disturbances.

Our aim is that of presenting a comparison between two types of computational approaches: those that provide a solution that is robustly guaranteed, and randomizedbased methods, which provide a solution with probabilistic guarantees only. We anticipate that the main outcome of the analysis is that the first type of approaches may show some conservatism in that they require the constraints to be loose in order to be feasible, while, although robustness cannot be a-priori guaranteed, randomized methods may lead to an effective solution - whose robustness can be experimentally verified - even for tight constraints. This enhances the use of randomized-based methods as a valid alternative to other approaches to robust MPC.

We consider the problem of setting up a robust MPC scheme for an uncertain discrete time linear system, whose state $x \in \mathbb{R}^{n}$ evolves according to the equation:

$$
x_{t+1}=A x_{t}+B u_{t}+w_{t}
$$

where $u \in \mathbb{R}^{m}$ is a control input while $w_{t}$ is an additive stochastic disturbance whose probability distribution has bounded support $W$. To be more concrete, we shall con-

\footnotetext{
* Research supported by the European Commission under the MoVeS project, FP7-ICT-2009-257005.
}

sider the case that $W$ is a polytope. We assume that the state of the system is available.

MPC amounts to optimizing a finite horizon cost with respect to a chosen control policy, taking into account both saturation constraints on the input and safety constraints on the state. Then, according to the well-known receding horizon strategy, just the first value of the so computed control action is actually implemented, and optimization is repeated at each time instant.

As for the finite horizon control problem to be solved at every time instant $\tau$, we suppose to minimize the average quadratic cost

$$
J=\mathbb{E}\left[\sum_{i=1}^{M} x_{t+i}^{T} Q x_{t+i}+\sum_{i=0}^{M-1} u_{t+i}^{T} R u_{t+i}\right],
$$

with the constraint that the state and the input stay at every time instant in the prediction horizon $M$ in the polytopic sets $\mathcal{X}$ and $\mathcal{U}$, respectively:

$$
\begin{aligned}
& x_{\tau+i} \in \mathcal{X} i=1, \ldots, M \\
& u_{\tau+i} \in \mathcal{U} i=0, \ldots, M-1 .
\end{aligned}
$$

The main issue with MPC in the present setup is how to deal with uncertainty. When the constraints (3) are enforced for every possible realization of the disturbance (robust MPC), the ensuing optimization problem turns out to be semi-infinite, i.e. a problem with a finite number of decision variables but an infinite amount of constraints. Semi-infinite problems require some care, since they may be very tough to solve, and in many cases they have been proven to be NP-hard.

A possible solution to this issue is that adopted in Mayne et al. [2005], Goulart et al. [2006], Rakovic et al. [2012], Evans et al. [2012b], where the uncertain evolution of 
the system (1) is properly over-bounded so that an optimization problem that it is amenable of resolution at a relatively low computational burden can be formulated. In this case, the obtained solution is guaranteed to robustly satisfy the constraints. Yet, the introduced overapproximation may introduce some conservatism, so that feasibility is achieved only if the constraints to be satisfied are loose.

Alternatively, one can opt for an inner approximation achieved through randomization - of the uncertain evolution of the system, and adopt the scenario-based MPC, Prandini et al. [2012], Deori et al. [2013], Calafiore and Fagiano [2013]. The paradigm is quite different from that of previous methods. In particular, the solution of the scenario-based MPC is not guaranteed to satisfy the constraints for all possible realizations of the disturbance, but the probability of violating the constraints at any step is guaranteed to be smaller than an user-chosen threshold $\varepsilon$ (chance-constrained solution). The scenariobased MPC was originally introduced for solving problems where achieving robustness is not feasible and a chanceconstrained reformulation is needed because of the unboundedness of the disturbance support. However, given that the smaller the threshold chosen by the user, the closer the scenario solution is to the robust one, the scenario approach can be also used as a heuristic method to find approximate (relaxed) solutions to robust MPC problems when the support of the disturbance is bounded. The advantage of a scenario-based solution is that it does not introduce any sort of conservatism in the constrained optimization problem resolution. It may hence lead to an effective solution even when constraints are tight and other approaches turn out to be unfeasible. This is shown in this paper, whose objective is that of performing a comparative analysis of the two types of approaches by means of prototype examples. The analysis highlights advantages and drawbacks of the various approaches, and all the results are substantiated by quantitative evaluations. As already anticipated, the performed analysis shows that scenariobased MPC may be a valid alternative to other approaches, enhancing its use to tackle robust MPC.

The rest of the paper is organized as follows. A review of two existing methods to robust MPC providing robustly guaranteed solutions is given in Section 2, while Section 3 presents an approach settled in the scenario-based MPC category. The performances of these three approaches are then evaluated on simulation examples in Section 4, while Section 5 provides a final discussion, including some conclusions.

\section{APPROACHES TO ROBUST MPC}

\subsection{Robust MPC based on invariant sets}

This first approach was originally proposed in Mayne et al. [2005] and it relies on invariant sets to bound the uncertain dynamics of (1).

The control law is selected as

$$
u_{\tau+i}=K\left(x_{\tau+i}-\bar{x}_{\tau+i}\right)+c_{i},
$$

where $K$ is an a-priori fixed gain (e.g. the optimal LQ gain of the infinite horizon problem without constraints), while the open loop term $c_{i}$ is the actual decision variable and $\bar{x}_{\tau+i}$ is the state variable of the following auxiliary nominal system

$$
\bar{x}_{\tau+i+1}=A \bar{x}_{\tau+i}+B c_{i},
$$

whose initialization $\bar{x}_{\tau}$ is a decision variable too. Given (4), the true system dynamics becomes

$$
x_{\tau+i+1}=(A+B K) x_{\tau+i}-B K \bar{x}_{\tau+i}+B c_{i}+w_{\tau+i},
$$

so that the difference between the actual and the nominal dynamics $\eta=x-\bar{x}$ satisfies the equation

$$
\eta_{\tau+i+1}=(A+B K) \eta_{\tau+i}+w_{\tau+i} .
$$

Now, let $Z$ be a disturbance invariant set for the system (5), that is, if $\eta_{t} \in Z$ then $\eta_{t+1}$ must belong to $Z$ too, for every realization of the disturbance $w_{t} \in W$. Based on the properties of invariant sets, it is trivial to verify that if $x_{\tau} \in \bar{x}_{\tau}+Z$ (note that $b+A=\{b+a, a \in A\}$ ), then $x_{\tau+i} \in \bar{x}_{\tau+i}+Z, i=1, \ldots, M$, and $u_{\tau+i} \in c_{\tau+i}+K Z$ (note that $K A=\{K a, a \in A\}$ ), $i=0, \ldots, M-1$. In other words, $Z$ can be used to bound the uncertain dynamics of (1). The robust satisfaction of constraints (3) can be guaranteed by selecting $\bar{x}_{\tau}$ so that $x_{\tau} \in \bar{x}_{\tau}+Z$, and $c_{i}$ so that $\bar{x}_{\tau+i}+Z \subseteq \mathcal{X}$ and $c_{i}+K Z \subseteq \mathcal{U}$. This leads to the following optimization problem:

$$
\begin{aligned}
& \min _{c_{i}, \bar{x}_{\tau}} \mathbb{E}\left[\sum_{i=1}^{M} x_{\tau+i}^{T} Q x_{\tau+i}+\sum_{i=0}^{M-1} u_{\tau+i}^{T} R u_{\tau+i}\right] \text { subject to: } \\
& \begin{cases}c_{i} \in \mathcal{U} \ominus K Z & i=0 \ldots M-1 \\
\bar{x}_{\tau+i} \in \mathcal{X} \ominus Z & i=1 \ldots M \\
x_{\tau}-\bar{x}_{\tau} \in Z\end{cases}
\end{aligned}
$$

where $\ominus$ denotes the Pontryagin difference $(A \ominus B=\{a$ : $a+B \subseteq A\})$.

If the invariant set $Z$ is a polytope, then the sets $\mathcal{U} \ominus K Z$ and $\mathcal{X} \ominus Z$ are polytopes too and can be easily computed. In this case, problem (6) is thus convex and can be solved through standard optimization techniques like those used in CVX, Grant and Boyd [2011], and YALMIP, Löfberg [2004].

The constraints in (6) can be seen as tightened versions of the original state and input constraints because of the difference with $Z$ and $K Z$. In order to achieved the widest feasibility for problem (6), the invariant set $Z$ should be as small as possible. The minimal invariant set, however, may not be a polytope, see Mayne et al. [2005], and moreover it is quite difficult to compute. Usually a polytopic outer approximation of the minimal invariant set is used, see e.g. Rakovic et al. [2005].

\subsection{Tube-based robust $M P C$}

This second approach was developed in Evans et al. [2012b] for the case of multiplicative uncertainty. The case of additive uncertainty discussed here is obtained by means of straightforward modifications.

Let $z=\mathbb{E}[x]$ and $e=x-\mathbb{E}[x]$, and select the control law as

$$
u_{\tau+i}=K z_{\tau+i}+L e_{\tau+i}+c_{i},
$$

where $K$ and $L$ are a-priori fixed gains and the open loop term $c_{i}$ is the actual decision variable. The dynamics of $x=z+e$, then, is split into the dynamics of $z$ (nominal dynamics) and of $e$ (uncertainty dynamics): 


$$
\begin{array}{ll}
z_{\tau+i+1}=(A+B K) z_{\tau+i}+B c_{i}, & z_{\tau}=x_{\tau} \\
e_{\tau+i+1}=(A+B L) e_{\tau+i}+w_{\tau+i}, & e_{\tau}=0 .
\end{array}
$$

The idea is to find at each time step in the prediction horizon of length $M$ a polytope $P_{\tau+i}$ enclosing any possible evolution of $e$ at time $\tau+i$ as due to the disturbance $w$. Then, the evolution of $x$ is guaranteed to be contained in $z_{\tau+i}+P_{\tau+i}$, while $u_{\tau+i}$ is contained in $K z_{\tau+i}+c_{i}+L P_{\tau+i}$ and the satisfaction of (3) is guaranteed by requiring that these polytopes are subsets of $\mathcal{X}$ and $\mathcal{U}$, respectively. The final optimization problem is

$$
\begin{aligned}
& \min _{c_{i}} \mathbb{E}\left[\sum_{i=1}^{M} x_{\tau+i}^{T} Q x_{\tau+i}+\sum_{i=0}^{M-1} u_{\tau+i}^{T} R u_{\tau+i}\right] \text { subject to: } \\
& \begin{cases}K z_{\tau+i}+c_{i} \in \mathcal{U} \ominus L P_{\tau+i} & i=0, \ldots, M-1 \\
z_{\tau+i} \in \mathcal{X} \ominus P_{\tau+i} & i=1, \ldots, M\end{cases}
\end{aligned}
$$

As for the computation of the bounding polytopes, $P_{\tau+i}$ are selected in the form $\left\{e_{\tau+i}: V e_{\tau+i} \leq \alpha_{i}\right\}$, where inequality is intended componentwise. The matrix $V$ has to be a-priori fixed so that the polytope facets have always the same orientation for every $t+i$. The bounding of the evolution of $e$ is achieved by suitably distancing the facets at each time instant as specified by vector $\alpha_{i}$. Technically speaking, the polytopes are recursively computed. Starting from $P_{\tau}=\{0\}, P_{\tau+i+1}$ is obtained from $P_{\tau+i}$ by imposing that the set of points $e_{\tau+i}$ whose evolution $e_{\tau+i+1}$ according to (9) is contained in $P_{\tau+i+1}$ must include the points in $P_{\tau+i}$, that is

$$
\begin{aligned}
& \left\{e_{\tau+i}: V e_{\tau+i} \leq \alpha_{i}\right\} \subseteq \\
& \left\{e_{\tau+i}: V(A+B L) e_{\tau+i}+V w_{\tau+i} \leq \alpha_{i+1}, \forall w_{\tau+i} \in W\right\}
\end{aligned}
$$

(11) can be enforced exploiting a corollary of Farkas's lemma, originally proven in Bitsoris [1988], about the inclusion of polytopes: let $\mathcal{S}_{1}=\left\{x: A_{1} x \leq b_{1}\right\}$ and $\mathcal{S}_{2}=\left\{x: A_{2} x \leq b_{2}\right\}$; it holds that $\mathcal{S}_{1} \subseteq \mathcal{S}_{2}$ if and only if there exists a matrix $H$ with non-negative entries such that $H A_{1}=A_{2}$ and $H b_{1} \leq b_{2}$.

In view of this lemma, (11) is equivalent to

$$
\left\{\begin{array}{lc}
H V=V(A+B L) & h_{k l} \geq 0 \quad \forall k, l \\
H \alpha_{i} \leq \alpha_{i+1}-V v & \forall v \text { vertex of } W
\end{array}\right.
$$

from which the polytopes $P_{\tau+i}$ are eventually determined by first selecting the $H$ satisfying (12a) that gives the minimum value for the trace of $H H^{T}$, and then by selecting the $\alpha_{i}$ with minimal components that satisfies (12b). Note that the inequality in (12b) is posed for the vertices of $W$ only thanks to convexity.

\section{SCENARIO-BASED MPC}

In the considered scenario-based solution, the control law is expressed as

$$
u_{\tau+i}=K x_{\tau+i}+c_{i},
$$

where $K$ is an a-priori fixed gain and the open loop term $c_{i}$ is the actual decision variable.

In order to determine the open loop term $c_{i}$, a number $N$ of disturbance realizations of length $M$

$$
w_{\tau}^{(k)}, w_{\tau+1}^{(k)}, \ldots, w_{\tau+M-1}^{(k)}, \quad k=1,2, \ldots, N
$$

are generated according to the underlying probability distribution of $w$. Then, a finite optimization problem, where the state and input constraints are posed in correspondence of the extracted realizations of the disturbance only, is considered.

To be precise, let $u_{\tau}^{(k)}, u_{\tau+1}^{(k)}, \ldots, u_{\tau+M-1}^{(k)}$ be the control actions evaluated in correspondence of the $k$-th extracted realization of the disturbance, and $x_{\tau+1}^{(k)}, x_{\tau+2}^{(k)}, \ldots, x_{\tau+M}^{(k)}$ the corresponding state trajectory (clearly, $u_{\tau+i}^{(k)}$ and $x_{\tau+i}^{(k)}$ still depend on the choice of $\left.c_{l}, l=1, \ldots, i\right)$. The scenario program to be solved at each step is

$$
\min _{c_{i}} \mathbb{E}\left[\sum_{i=1}^{M} x_{\tau+i}^{T} Q x_{\tau+i}+\sum_{i=0}^{M-1} u_{\tau+i}^{T} R u_{\tau+i}\right] \text { subject to: }
$$

$$
\left\{\begin{array}{ll}
u_{\tau+i}^{(k)} \in \mathcal{U} & i=0, \ldots, M-1 \\
x_{\tau+i}^{(k)} \in \mathcal{X} & i=1, \ldots, M
\end{array}, k=1, \ldots, N .\right.
$$

Because of the finiteness of the considered realizations of the disturbance, problem (14) has a finite number of constraints only and is convex. Indeed, using the parametrization (13), both the input and the state depend linearly on the parameters $c_{i}$, and this entails that: $\left.i\right)$ the cost function is convex in the decision variables (actually, it is a quadratic function); and ii) for every fixed realization of the disturbance, the constraints (3) are convex as well (when $\mathcal{U}$ and $\mathcal{X}$ are polytopes, they are linear).

Despite the apparent naivety of the scenario approach, the obtained solution comes with some interesting guarantees about constraint feasibility, Calafiore and Campi [2005, 2006], Campi and Garatti [2008], Campi et al. [2009], Alamo et al. [2009], which make it a sensible method to find an approximate solution to a robust problem, as discussed in the introduction. In the present convex set-up, the best available result is given by the following theorem. Theorem 1. Let $r$ be the total number of optimization variables in problem (14). For any $\varepsilon \in(0,1)$ and $\beta \in(0,1)$, if

$$
N \geq \frac{r+1+\ln (1 / \beta)+\sqrt{2(r+1) \ln (1 / \beta)}}{\varepsilon},
$$

then, the probability that there exists a disturbance realization such that the solution to (14) does not satisfy the constraint

$$
\begin{cases}u_{\tau+i} \in \mathcal{U} & i=0, \ldots, M-1 \\ x_{\tau+i} \in \mathcal{X} & i=1, \ldots, M\end{cases}
$$

is no bigger than $\varepsilon$ with high confidence $1-\beta$.

The proof can be found in Campi and Garatti [2008], where, however, an implicit expression for the bound on $N$ is given. The explicit expression used here is due to Alamo et al. [2010].

In words, Theorem 1 says that the solution provided by the scenario approach is robust except for an $\varepsilon$ portion of the disturbance realizations ( $\varepsilon$-robustness), as long as $N$ is suitably chosen. Note that $\varepsilon$-robustness is guaranteed with high confidence $1-\beta$ only. However, if one adopts small values of $\beta$ like $\beta=10^{-6}$ or $\beta=10^{-9}$, then, the $\varepsilon$-robustness is achieved beyond any reasonable doubt.

\section{COMPARATIVE ANALYSIS}

The goal of this section is to make a comparative analysis of the approaches described in Sections 2 and 3, focusing 
in particular on their capability of providing an effective solution to the MPC problem as the state and input constraints become tighter and tighter. To this purpose, the approaches are applied in a receding horizon fashion to a second order toy system, first, and, then, to a fourth order spring-mass system.

\subsection{Second order system}

We consider a second order system described by:

$$
x_{t+1}=\left[\begin{array}{cc}
0.5 & -0.5 \\
0.5 & 0.5
\end{array}\right] x_{t}+\left[\begin{array}{l}
1 \\
0
\end{array}\right] u_{t}+w_{t},
$$

where $w$ is a white noise uniformly distributed in $\left[\begin{array}{ll}-0.2 & 0.2\end{array}\right]^{2}$. The reference finite horizon problem takes the following form:

$$
\begin{aligned}
& \min \mathbb{E}\left[\sum_{i=1}^{M} x_{\tau+i}^{T} Q x_{\tau+i}+\sum_{i=0}^{M-1} u_{\tau+i}^{T} R u_{\tau+i}\right] \text { subject to: } \\
& \begin{cases}\left\|u_{\tau+i}\right\|_{\infty} \leq \bar{u} \quad i=0, \ldots, M-1 \\
\left\|C x_{\tau+i}\right\|_{\infty} \leq \bar{y} & i=1, \ldots, M\end{cases}
\end{aligned}
$$

where we set

$$
M=10, \quad Q=I_{2}, \quad R=0.1, \quad C=I_{2} .
$$

As for the design parameters entering the solution of (16) according to the approaches of Sections 2 and 3:

- the feedback gains $K$ in (4), $K, L$ in (7), and $K$ in (13) are set equal to the optimal LQ gain $K_{L Q}$;

- the shaping matrix $V$ of the tube-based approach is

$$
V=\left[\begin{array}{cccccccc}
1 & 0 & -1 & 0 & 1 & 1 & -1 & -1 \\
0 & 1 & 0 & -1 & 1 & -1 & 1 & -1
\end{array}\right]^{T}
$$

- the violation and confidence parameters of the scenariobased approach are $\varepsilon=0.05$ and $\beta=10^{-6}$;

We set the initial state equal to 0 and evaluate the threshold values $\bar{y}_{T}$ and $\bar{u}_{T}$ for $\bar{y}$ and $\bar{u}$ in (16) leading to unfeasibility of the optimization problems (6), (10), and (14). Our aim is to find how much the constraints can be tightened before incurring in unfeasibility so as to assess the possible conservativeness of the three approaches. Results are shown in Table 1.

Table 1. Estimate of the threshold values $\bar{y}_{T}$ and $\bar{u}_{T}$ for $\bar{y}$ and $\bar{u}$ before incurring in unfeasibililty.

\begin{tabular}{r|rr} 
& $\bar{y}_{T}$ & $\bar{u}_{T}$ \\
\hline Tube-based approach & 0.74 & 0.46 \\
\hline Approach based on invariant sets & 0.53 & 0.35 \\
\hline Scenario-based approach & 0.44 & 0.30
\end{tabular}

Note that, when the initial state is zero, the optimization problem (6) in the approach of Section 2.1 is feasible if and only if the invariant set $Z$ and its projection $K Z$ on the input space through the feedback gain $K$ are respectively contained in the constraint set $\mathcal{X}$ and $\mathcal{U}$. Hence, the thresholds $\bar{y}_{T}$ and $\bar{u}_{T}$ can be obtained based on $Z$ and $K Z$. Likewise, when the initial state is zero, problem (10) in the approach of Section 2.2 is feasible if only if the tube sections $P_{\tau+i}$ and $L P_{\tau+i}$ are contained in the input and state constraint sets for every $i$. Since $P_{\tau+i}$ and $L P_{\tau+i}$ are increasing with $i, \bar{y}_{T}$ and $\bar{u}_{T}$ are obtained based on the tube sections $P_{t+M}$ and $L P_{t+M-1}$.

Computing $\bar{y}_{T}$ and $\bar{u}_{T}$ for the scenario-based approach is instead more tricky, since it is a randomized method. The values reported in Table 1 are heuristically determined by progressively reducing $\bar{y}$ and $\bar{u}$ and checking for each pair $(\bar{y}, \bar{u})$ whether problem (14) is feasible in 100 trials.

As it appears, the scenario-based approach outperforms the other approaches in terms of tightness allowed for the constraints. This can be justified by comparing the different approximations of the uncertain evolution of the state used by the three approaches. To this purpose, we take a bunch of 10000 disturbance realizations of length $M$ and simulate the state evolution from the initial condition $x_{0}=0$ when the control input is given by $u_{\tau+i}=$ $K_{L Q} x_{\tau+i}$. Indeed, this is the solution to problems (6), (10), and (14) when constraints are feasible and $x_{0}=0$. We obtain for every time instant along the prediction horizon $[0, M]$ a cloud of possible states reached by the system and we superimpose them to the invariant set $Z$ and to the polytopes $P_{\tau+i}$ defining the tube section at that time instant.

As one can see in Fig.1 the clouds of reachable states are
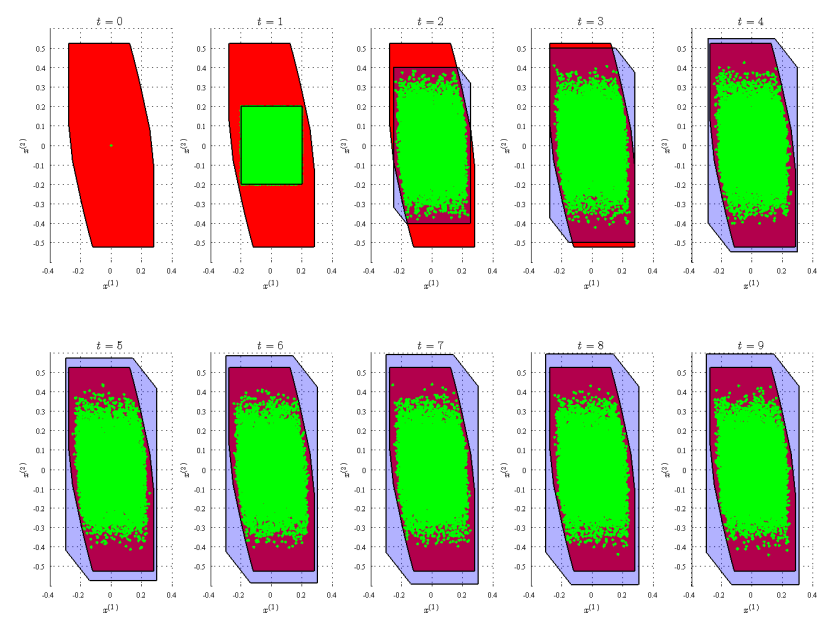

Fig. 1. Realizations of the state of system (15) when the LQ control law is applied (green cloud), invariant set $Z$ (red) and sections of the tube (light blue).

smaller than their approximations as given by $Z$ and $P_{t+i}$. In particular note that the invariant set $Z$ has to contain the cloud at every time instant and it cannot adapt to the shape of the cloud. Furthermore, even if we superimpose all the clouds, some regions of the invariant set appear empty and hence are quite unlikely to be reached. Using tubes gives the possibility to shape the reachable set approximation so as to best fit the cloud, but it turns out that the tube approximation is well adapted to the cloud just for the first 4 time steps and then becomes even larger than the invariant set. The scenario-based approach, instead, is not affected by this over approximation effect. As a matter of fact, according to problem (14) the optimal control law is determined by considering $N$ disturbance realizations and the corresponding state and input values for defining the constraints. The resulting approximation of the un- 
certain state evolution is then tighter then that used by the other two approaches. Indeed, since we adopted the parametrization $u_{t+i}=K_{L Q} x_{t+i}+c_{i}$ for the scenariobased approach, the clouds plotted in Fig. 1 represent the reachable states for the scenario solution.

Note that the feasibility of problems (6), (10) and (14) depends on the choice of the feedback gains $K$ in (4), $K$, $L$ in (7), and $K$ in (13). It may be that choices other than $K_{L Q}$ lead to smaller thresholds than those reported in Table 1 . However, in (6) and (10) $K$ and $(K, L)$ have to be fixed in advance, otherwise computational difficulties arise, and it is usually difficult to guess what is the right choice. In the scenario method, instead, the feedback gain $K$ can be easily optimized along with the open loop term $c$, so that $K$ can be automatically tuned towards the optimum, ?. To be precise, to preserve convexity, one has to adopt the following parametrization

$$
u_{t+i}=\gamma_{i}+\sum_{j=0}^{i-1} \theta_{i, j} w_{t+j},
$$

where $w_{t+j}$ can be reconstructed from the state equation as $x_{t+j+1}-A x_{t+j}-B u_{t+j}$ and $\theta_{i, j} \in \mathbb{R}^{m \times n}$ and $\gamma_{i} \in \mathbb{R}^{m}$ are the design parameters. It can be proven that for any control law $u_{t+i}=K_{i} x_{t+i}+c_{i}$, there exists a suitable choice of $\theta_{i, j}$ and $\gamma_{i}$ such that the control action $u_{t+i}$ is the same in the two cases, and viceversa, Goulart et al. [2006]. Hence, (17) is equivalent to optimizing both the open loop term and the feedback gain, and by using it a further improvement in terms of tightness of the bounds can be achieved. For instance, where a reduced parametrization is adopted where the control input depends only on the previous 3 values of the disturbance, then, the threshold values $\bar{y}_{T}=0.39$ and $\bar{u}_{T}=0.28$ are obtained.

As for the receding horizon implementation of the three approaches, when constraints are loose and they are all feasible, the obtained performance are quite similar. More specifically, after some transient, they all converge to the LQ solution, also when using the control law parametrization (17) for the scenario-based approach. When constraints are tight and only the scenario-based approach with parametrization (17) provides a feasible solution, then such a solution does not necessarily converge to the LQ one. This appears to be the case if we set $\bar{u}=0.28$ and $\bar{y}=0.39$, as shown in Fig. 2, where a realization of the control input and the corresponding values as given by the LQ control law are plotted.

\subsection{Spring-mass system}

We next present the simulation results obtained applying the three approaches to a simple mechanical system composed of two masses and two springs, as shown in Fig.3. The 4-dimensional state of the system is given by mass displacements with respect to an equilibrium point and their derivatives, whereas the control input is given by two forces acting on the masses. The system is discretized with standard techniques assuming constant input between consecutive time steps. All stiffness and masses are set equal to 1 . The disturbance added to the discrete time state is uniformly distributed in $[-1]^{4}$. The finite horizon control problem takes the form (16) with the following parameter setting:

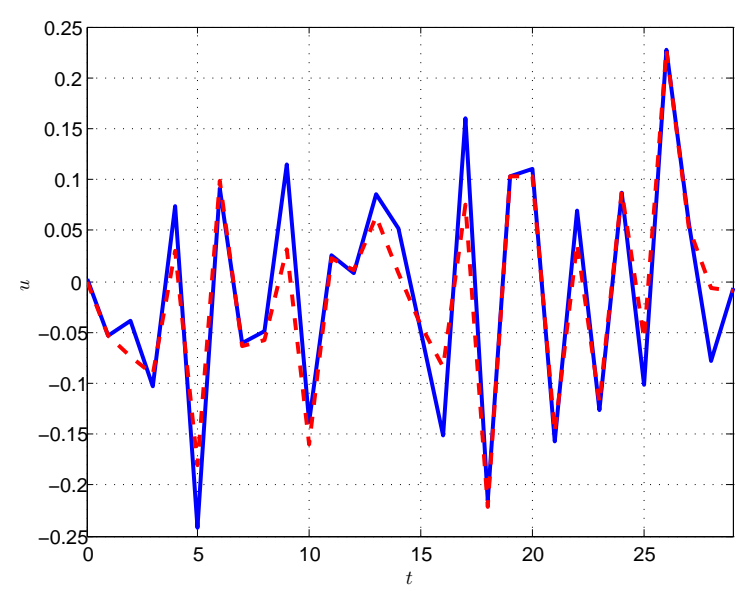

Fig. 2. Input for system (15) controlled with the scenariobased approach applied in a receding horizon fashion (solid blue) and input given by the LQ control law (dashed red).

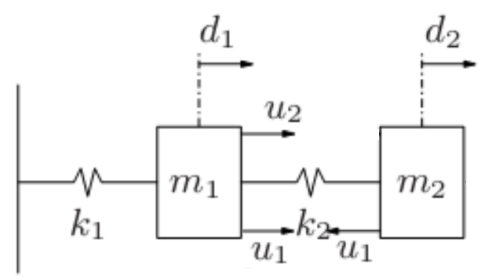

Fig. 3. Scheme of the mechanical system.

$$
\begin{gathered}
Q=\left[\begin{array}{cc}
I_{2} & 0 \\
0 & 0
\end{array}\right] \quad R=I_{2} \quad M=5 \\
C=\left[\begin{array}{cccc}
1 & 0 & 0 & 0 \\
-1 & 1 & 0 & 0
\end{array}\right] .
\end{gathered}
$$

In particular, $C$ appearing in the state constraints is chosen so that $\bar{y}$ in (16) represents some bound posed on the deformation of both springs. The shape of the tube sections used in the approach of Section 2.2 is a 4dimensional hyper-rectangle: $V=\left[I_{4}-I_{4}\right]^{T}$.

As in the previous example, we first determine how much we can tighten the constraints before reaching unfeasibility for the three approaches. Also in this case the scenario approach is still feasible for much tighter constraints than the other two approaches (see Table 2), the gap being larger when the feedback gain is optimally tuned by using the parametrization in (17). The tube-based approach turns

Table 2. Estimate of the threshold values $\bar{y}_{T}$ and $\bar{u}_{T}$ for $\bar{y}$ and $\bar{u}$ before incurring in unfeasibililty.

\begin{tabular}{r|cc} 
& $\bar{y}_{T}$ & $\bar{u}_{T}$ \\
\hline Tube-based approach & 23 & 20 \\
\hline Approach based on invariant sets & 5.2 & 4.2 \\
\hline Scenario-based approach with $K=K_{L Q}$ & 3.5 & 2.5 \\
\hline Scenario-based approach with optimally tuned $K$ & 2.8 & 1.7
\end{tabular}

out to be much more conservative than the approach based on invariant sets, and this result is further confirmed by the 
plots in Fig. 4 where the approximations of the uncertain system dynamics of the two approaches are compared.

Similarly to the previous example, if we take loose constraints and apply the three approaches in a receding horizon fashion, they all converge to the LQ gain. Still, for tight constraints $(\bar{y}=2.8$ and $\bar{u}=1.7)$ the scenariobased approach optimizes the state feedback gain through an appropriate choice of the $\theta$ and $\gamma$ parameters in (17) so as to remain feasible (see Fig. 5).
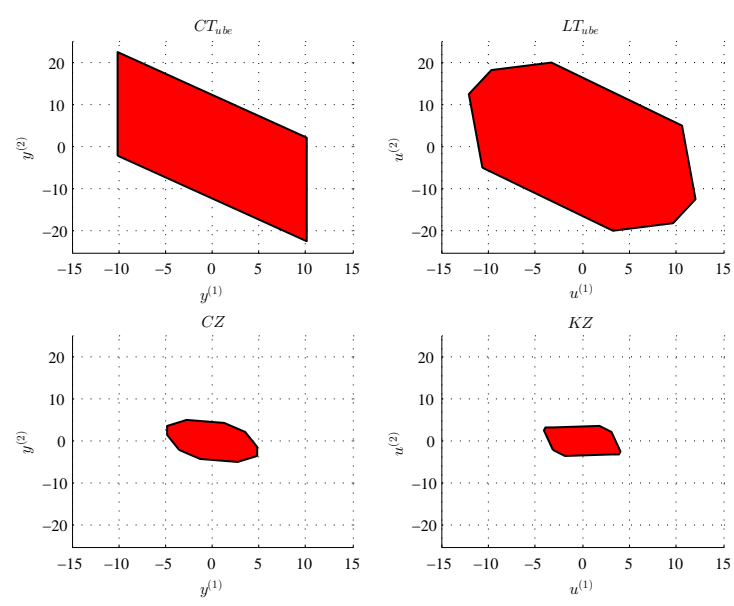

Fig. 4. Projections of the largest section of the tube (top row) and of the invariant set $Z$ (bottom row) on the output space (left plot) and on the input space (right plot).

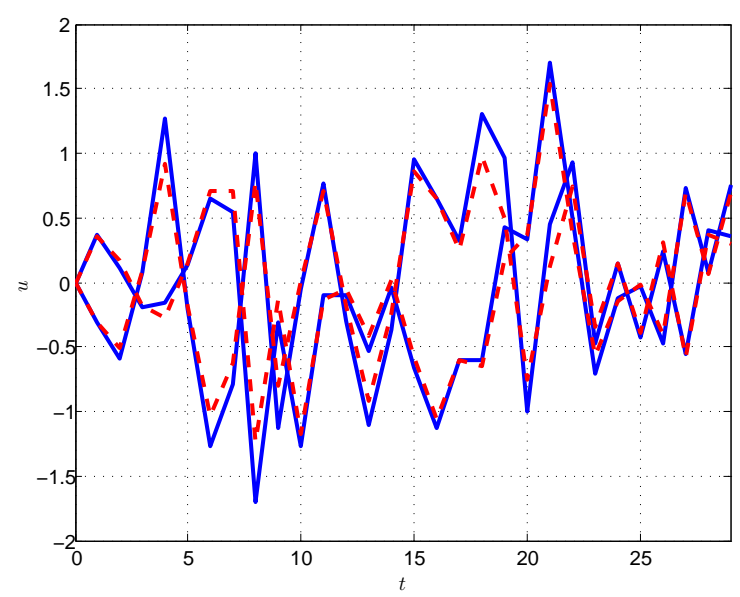

Fig. 5. Control input obtained with the scenario-based approach applied in a receding horizon fashion (blue) and input given by the LQ control law (dashed red).

These results confirm the ones in the previous example and spot out even a larger gap between the limit of feasibility of the three approaches. This is most likely due to the increased dimensionality of the system: the outer approximations of the uncertain system dynamics given by the invariant sets or by the tubes are adversely affected by the dimension of the system and become more conservative, while the inner approximation used by the scenario-based approach is not influenced by the system dimensionality and its probabilistic guarantees remain valid.

\section{FINAL DISCUSSION}

The results of the comparative analysis in Section 4 highlight some key features of the approaches in Sections 2 and 3. Specifically, the approaches in Section 2 may not be applicable to problems with tight constraints. Unfeasibility may in fact arise due to their use of $i$ ) an outer approximation of the uncertain system dynamics, and ii) a-priori fixed feedback gains in the control law parametrization. On the contrary, the scenario-based approach is able to tackle problems with tighter constraints because of the adopted inner approximation and, possibly, of the tuning of the state feedback gain. Both numerical examples reveal in fact that, though setting the feedback gain to the LQ gain is an optimal choice for what concerns the cost minimization, it may be not 'optimal' for the constraints satisfaction.

In turn, however, while the approaches in Section 2 are robust in that constraints are guaranteed to be satisfied for every and each disturbance realization, for the scenariobased approach only probabilistic guarantees are given. This drawback of the scenario approach can be only partly alleviated by setting the size $\varepsilon$ of the set of disturbance realizations that violate the constraints as small as possible, compatibly with the available computational resources.

As for the receding horizon implementation of the approaches in Section 2, recursive feasibility and stability results have been developed in the literature (see e.g. Mayne et al. [2005, 2000] and Cannon et al. [2009], Evans et al. [2012a]). Results on these two issue have still to be worked out for the scenario-based approach. In particular, recursive feasibility is an open issue when state constraints are present, whereas it is easily shown if only input constraints are given (just set to zero the $\theta$ parameters in (17)). As for stability of the scenario-based infinite horizon implementation, BIBO stability is easily shown when the system is stable and the control input is subject to saturation constraints, but otherwise is still an open topic of research.

\section{REFERENCES}

T. Alamo, R. Tempo, and E. F. Camacho. A randomized strategy for probabilistic solutions of uncertain feasibility and optimization problems. IEEE Transactions on Automatic Control, 54(11):2545-2559, 2009.

T. Alamo, R. Tempo, and A. Luque. On the sample complexity of randomized approaches to the analysis and design under uncertainty. In Proceedings of the 2010 American Control Conference, pages 4671-4676, Baltimore, MD, USA, 2010.

Georges Bitsoris. On the positive invariance of polyhedral sets for discrete-time systems. Systems $\&$ control letters, 11(3):243-248, 1988.

G. Calafiore and M.C. Campi. Uncertain convex programs: randomized solutions and confidence levels. Mathematical Programming, 102(1):25-46, 2005.

G. Calafiore and M.C. Campi. The scenario approach to robust control design. IEEE Transactions on Automatic Control, 51(5):742-753, 2006.

G. Calafiore and L. Fagiano. Robust model predictive control via scenario optimization. IEEE Transactions on Automatic Control, 58:219-224, 2013. 
M.C. Campi and S. Garatti. The exact feasibility of randomized solutions of uncertain convex programs. SIAM Journal on Optimization, 19(3):1211-1230, 2008.

M.C. Campi, S. Garatti, and M. Prandini. The scenario approach for systems and control design. Annual Reviews in Control, 33(2):149-157, 2009.

Mark Cannon, Basil Kouvaritakis, and Desmond Ng. Probabilistic tubes in linear stochastic model predictive control. Systems \&6 Control Letters, 58(10):747-753, 2009.

Luca Deori, Simone Garatti, and Maria Prandini. Stochastic constrained control: trading performance for state constraint feasibility. In Proceedings of the 2013 European Control Conference, 2013.

Martin Evans, Mark Cannon, and Basil Kouvaritakis. Linear stochastic mpc under finitely supported multiplicative uncertainty. In Proceedings of the 2012 American Control Conference, 2012a.

Martin Evans, Mark Cannon, and Basil Kouvaritakis. Robust mpc for linear systems with bounded multiplicative uncertainty. In Proceedings of the 51st IEEE Conference on Decision and Control, 2012b.

P.J. Goulart, E.C. Kerrigan, and J.M. Maciejowski. Optimization over state feedback policies for robust control with constraints. Automatica, 42(4):523-533, 2006.

M. Grant and S. Boyd. CVX: Matlab software for disciplined convex programming, version 1.21 . http://cvxr.com/cvx, Feb 2011.

J. Löfberg. Yalmip: a toolbox for modeling and optimization in MATLAB. In Proceedings of 13th IEEE Symposium on Computer Aided Control System Design, 2004. URL http://control.ee.ethz.ch/ joloef/yalmip.php.

David Q Mayne, James B Rawlings, Christopher V Rao, and Pierre OM Scokaert. Constrained model predictive control: Stability and optimality. Automatica, 36(6): 789-814, 2000.

David Q Mayne, Maria M Seron, and SV Raković. Robust model predictive control of constrained linear systems with bounded disturbances. Automatica, 41(2):219-224, 2005.

Maria Prandini, Simone Garatti, and John Lygeros. A randomized approach to stochastic model predictive control. In Proceedings of the 51st IEEE Conference on Decision and Control, pages 7315-7320, 2012.

Sasa V Rakovic, Eric C Kerrigan, Koustas I Kouramas, and David Q Mayne. Invariant approximations of the minimal robust positively invariant set. IEEE Transactions on Automatic Control, 50(3):406-410, 2005.

S.V. Rakovic, B. Kouvaritakis, M. Cannon, C. Panos, and R. Findeisen. Parameterized tube model predictive control. IEEE Transactions on Automatic Control, 57: 2746-2761, 2012. 